\title{
Miliary Tuberculosis Mimicking Brain Metastasis from Renal Cell Carcinoma
}

\author{
Yousang Ko, MD', Eun-Kyung Mo, MD, PhD ${ }^{1}$, Yong Bum Park, $\mathrm{MD}^{1}$, Mi-Ri Kang, MD, MsC ${ }^{2}$, Jong Seok Bae, MD, PhD ${ }^{3}$, \\ Yerim Kim, MD, $\mathrm{MsC}^{3}$ \\ ${ }^{1}$ Department of Pulmonary and Critical Care Medicine, Kangdong Sacred Heart Hospital, Hallym University College of Medicine, Seoul; ${ }^{2}$ Department of Neurology, Busan \\ Paik Hospital, Inje University College of Medicine, Busan; ${ }^{3}$ Department of Neurology, Kangdong Sacred Heart Hospital, Hallym University College of Medicine, Seoul, \\ Korea
}

Background: Miliary tuberculosis (TB) can cause diagnostic confusion for clinicians because its radiological appearance can resemble that of metastatic cancer.

Case Report: Here, we describe the case of a 72-yearold woman with miliary TB mimicking brain metastasis from renal cell carcinoma. The patient visited our clinic because of dysarthria and sluggish speech. A metastatic cancer such as renal cell carcinoma or brain tumor was suspected. However, the patient was diagnosed with miliary TB associated with multiple intracranial tuberculomas and a subsequent paradoxical response to anti-TB therapy.

Conclusion: Clinicians should be aware that miliary TB can mimic metastatic cancer even in older people, especially in TB-endemic regions.

\section{J Neurocrit Care 2018;11(1):47-53}

Key words: Tuberculosis, Miliary; Tuberculoma, Intracranial; Brain neoplasms; Neoplasm metastasis

\author{
Received April 12, 2018 \\ Revised June 12, 2018 \\ Accepted June 15, 2018 \\ Corresponding Author: \\ Yerim Kim, MD, MsC \\ Department of Neurology, Kangdong \\ Sacred Heart Hospital, Hallym \\ University, College of Medicine, 150 \\ Seongan-ro, Gangdong-gu, Seoul \\ 05355, Korea \\ Tel: +82-2-2224-2854 \\ Fax: +82-2-488-0114 \\ E-mail: brainyrk@gmail.com
}

Copyright $\odot 2018$ The Korean Neurocritical Care Society

\section{INTRODUCTION}

Miliary tuberculosis (TB) is a rare form of TB, and data from the US Centers for Disease Control and Prevention in 2012 indicated that approximately 3.5\% of all cases of TB were classified as miliary TB. ${ }^{1}$ Furthermore, miliary TB can occur on the background of a previous malignancy, but also mimics metastatic cancer. ${ }^{2,3}$ Here, we report an interesting case of miliary TB mimicking brain metastasis from renal cell carcinoma.

\section{CASE REPORT}

A 72-year-old woman was referred to the neurosurgery department of our hospital because of abnormal findings on a brain computed tomography (CT). She had been healthy until two month before. Recently, the patient presents complaining of several weeks of generalized weakness and poor oral intake, when dysarthria and sluggish speech developed. She was a nonsmoker and denied using illicit drugs or other relevant medical history. She had no family history of TB and denied a contact with a person with TB infection. On examination, she appeared well:

cc This is an Open Access article distributed under the terms of the Creative Commons Attribution Non-Commercial License (http://creativecommons.org/licenses/by$\mathrm{nc} / 4.0$ ) which permits unrestricted non-commercial use, distribution, and reproduction in any medium, provided the original work is properly cited. 
body weight, $54 \mathrm{~kg}$; height, $160 \mathrm{~cm}$; temperature, $36.5^{\circ} \mathrm{C}$; blood pressure, $140 / 80 \mathrm{mmHg}$; pulse, 78 beats/min; respiration rate, 20/min; oxygen saturation, 99\% while breathing ambient air. The remainder of the examination was normal, including the neurologic examination. Laboratory results were normal. A human immunodeficiency virus (HIV) antibody test was negative.

A CT scan of the head, obtained after the administration of contrast material (Fig. 1) outside hospital, showed numerous small enhancing nodules with marked edema in both cerebral and cerebellar hemispheres and the brain stem. Next, brain magnetic resonance imaging (MRI) was performed. Axial T1- and T2-weighted contrast-enhanced MRI confirmed the results of the $\mathrm{CT}$ (Fig. 2A, B). Also, some abnormal restricted lesions were seen in diffusion-weighted imaging (Fig. 2C). A chest radiograph and a CT scan of the chest revealed uniform-sized small nodules randomly distributed throughout both lungs (Fig. 3). A lumbar puncture was performed. Cerebrospinal fluid analysis showed: opening pressure $125 \mathrm{mmH}_{2} \mathrm{O}$; color clear; $\mathrm{pH}$ 8.0; white blood cells negative; protein $33.7 \mathrm{mg} / \mathrm{dL}$ (normal, 10-50); glucose $81 \mathrm{mg} / \mathrm{dL}$ (blood glucose $122 \mathrm{mg} / \mathrm{dL}$ ); and adenosine deaminase 1.2 IU/L. Queckenstedt test demonstrated free cerebrospinal fluid (CSF) communication. CSF Gram, acid-fast bacilli (AFB) and India ink stains were negative.
Mycobacterium tuberculosis polymerase chain reaction (PCR) produced negative results. Initial sputum AFB was negative. An abdominal CT scan with contrast enhancement showed an ill-defined delayed-enhancing mass-like lesion involving the right lower kidney (Fig. 4A). Wholebody positron emission tomography/CT scan showed a diffuse, intensely hypermetabolic mass in the right kidney (Fig. 4B). A diagnosis of metastatic renal cell carcinoma was made, and the patient received two doses of radiotherapy for the purpose of reducing her neurologic symptoms, without improvement.

We performed bronchoalveolar lavage (BAL) and transbronchial lung biopsy (TBLB) at the anterior segment of the right upper lung. The next day, the microbiological investigation of BAL fluid by TB PCR gave a positive result, but there was no evidence of TB infection in the TBLB specimen. We therefore diagnosed the patient with miliary TB. One week after she visited the emergency room, she was treated with isoniazid, rifampicin, ethambutol, and pyrazinamide. She received intravenous dexamethasone $0.4 \mathrm{mg} / \mathrm{kg}$ per day and then tapered off. For the differential diagnosis of renal cell carcinoma, a renal biopsy was performed. The histopathological findings demonstrated chronic granulomatous inflammation with positive TB $P C R$, but acid-fast bacilli staining was negative (Fig. 5). Her
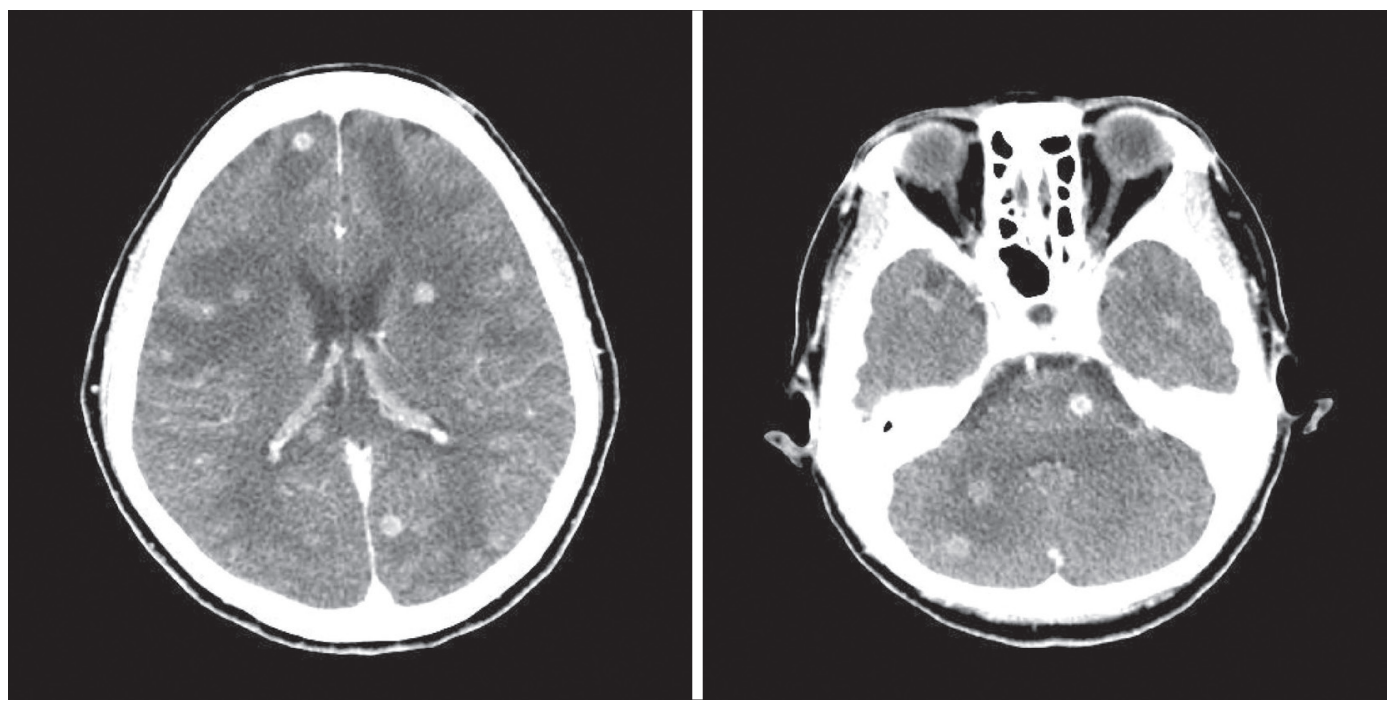

Figure 1. Axial images of contrast-enhanced computed tomography of the brain show numerous small enhancing masses with marked surrounding edema in both cerebral and cerebellar hemispheres and the brain stem. 

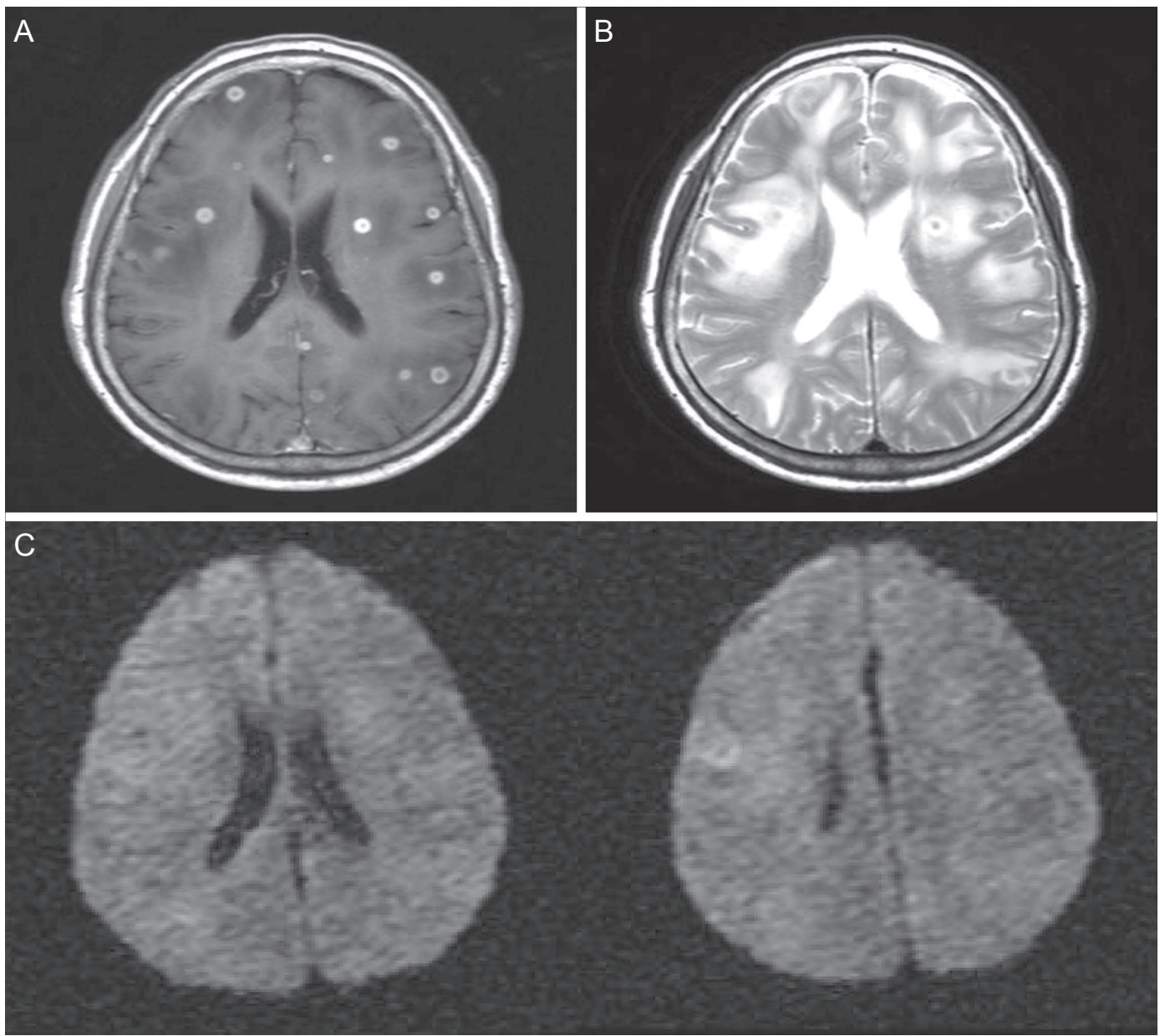

Figure 2. Magnetic resonance imaging of the brain on admission. (A, B) T1- and T2-weighted axial images, and an image with gadolinium enhancement. Multiple ring-enhancing lesions with surrounding edema are seen in both hemispheres. (C) Diffusion-weighted images. Some abnormal restricted lesions are seen in both hemispheres.
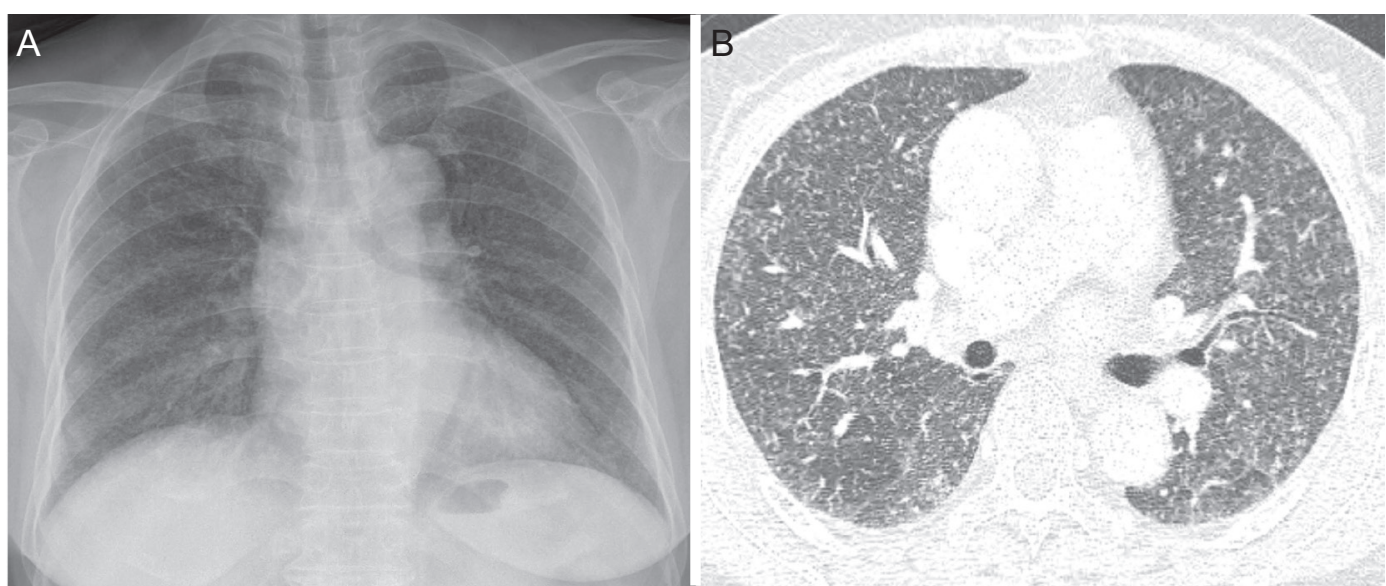

Figure 3. (A) Chest radiograph at the time of presentation shows diffuse micronodules involving both lung fields. (B) Chest computed tomography scan in the lung window reveals uniform-sized small nodules randomly distributed throughout both lungs. 

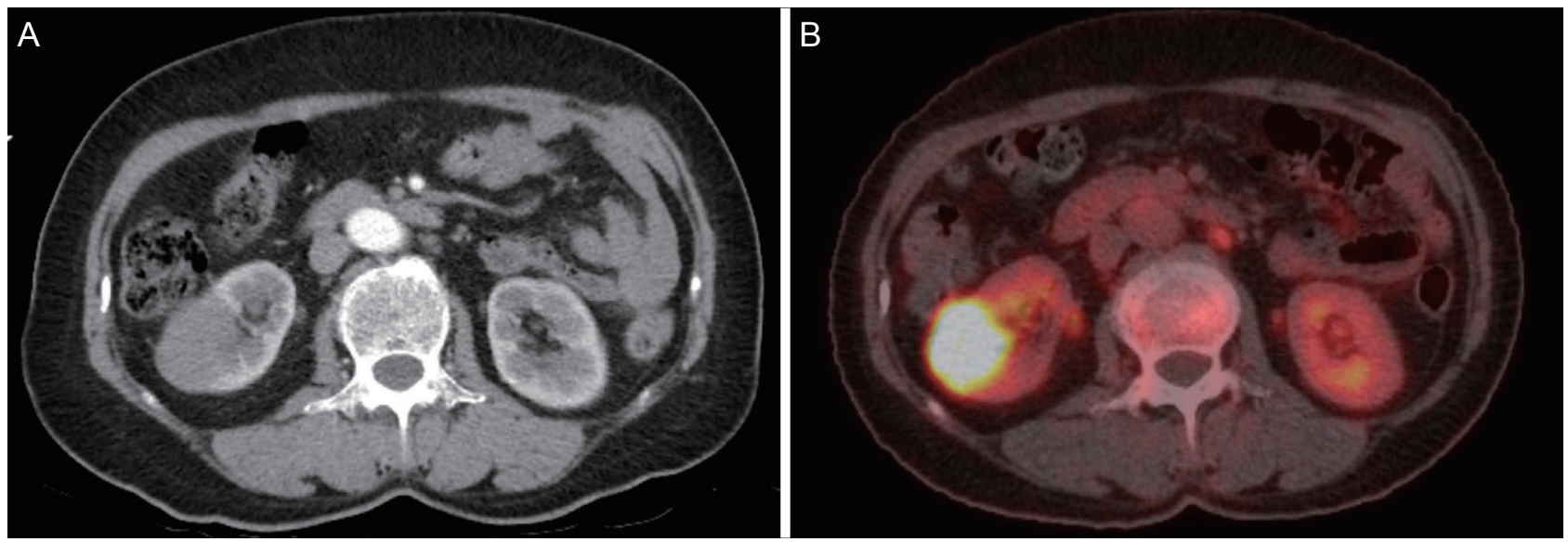

Figure 4. (A) Contrast-enhanced CT scan of the abdomen shows a $3.0 \times 4.5 \mathrm{~cm}$ enhancing mass in the right lower kidney. (B) PET/CT of a fused transaxial view shows high fluorodeoxyglucose uptake $\left(S_{U} V_{\max }=19.5\right)$ at the same site. $C T$, computed tomography; $P E T$, positron emission tomography; SUV ${ }_{\max }$, maximum standardized uptake value.

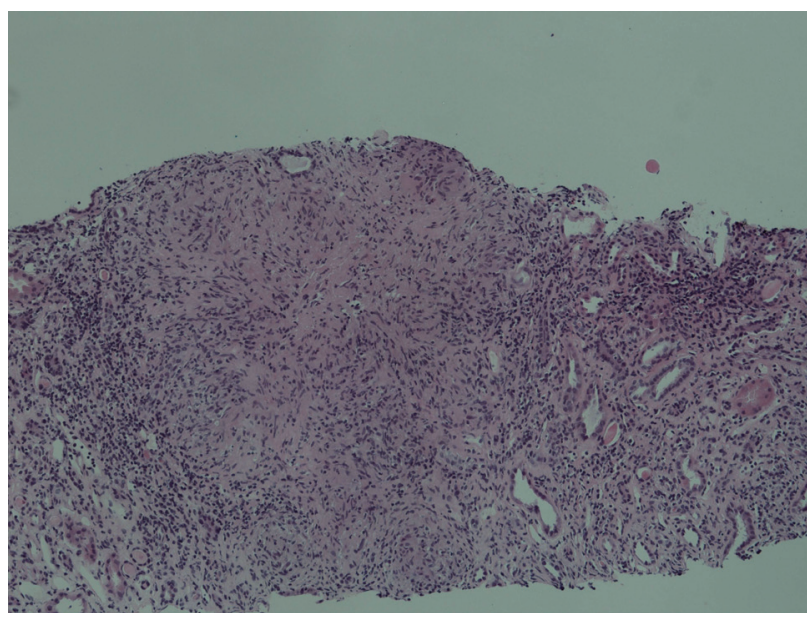

Figure 5. Percutaneous needle biopsy of the right kidney shows well-formed granulomas scattered in the interstitium of the medulla without obvious necrosis (hematoxylin and eosin staining, $\times 100)$.

symptoms began to improve one week after she received the anti-TB agents and she was discharged without complications.

Four weeks later, the patient returned to the hospital because of relapse of dysarthria and intermittent disorientation. The follow-up brain MRI showed no gross intervening changes in the numerous small rim-enhancing nodules and several new lesions had developed (Fig. 6A). To confirm that the diagnosis was accurate, a stereotactic brain biopsy was performed, but only reactive astrocytosis was observed, with negative results in TB PCR. Therefore, it was suggested that the patient be diagnosed with a paradoxical response, and corticosteroid was added to the treatment. After treatment for two more weeks, the patient's neurologic symptoms had improved. Mycobacterium tuberculosis was isolated from the BAL fluid and a solid media drug sensitivity test revealed no resistance to any anti-TB agent. A follow-up brain MRI after three months showed that both the multiple intracranial nodules and the surrounding edema had nearly disappeared (Fig. 6B). Finally, the patient was diagnosed with miliary TB associated with multiple intracranial tuberculoma with a subsequent paradoxical response to anti-TB therapy.

\section{DISCUSSION}

Although the World Health Organization Stop TB Strategy has helped to reduce the overall incidence of TB since 2004, TB remains a challenging problem in several countries, including developing countries such as South Korea. Miliary TB is considered to be extrapulmonary TB in the past, but classified as pulmonary TB because there are lesions in the lungs despite the multiplicity of organs affected. Because of its characteristic of multiorgan involvement, miliary TB can be a potentially lethal disease if it is 

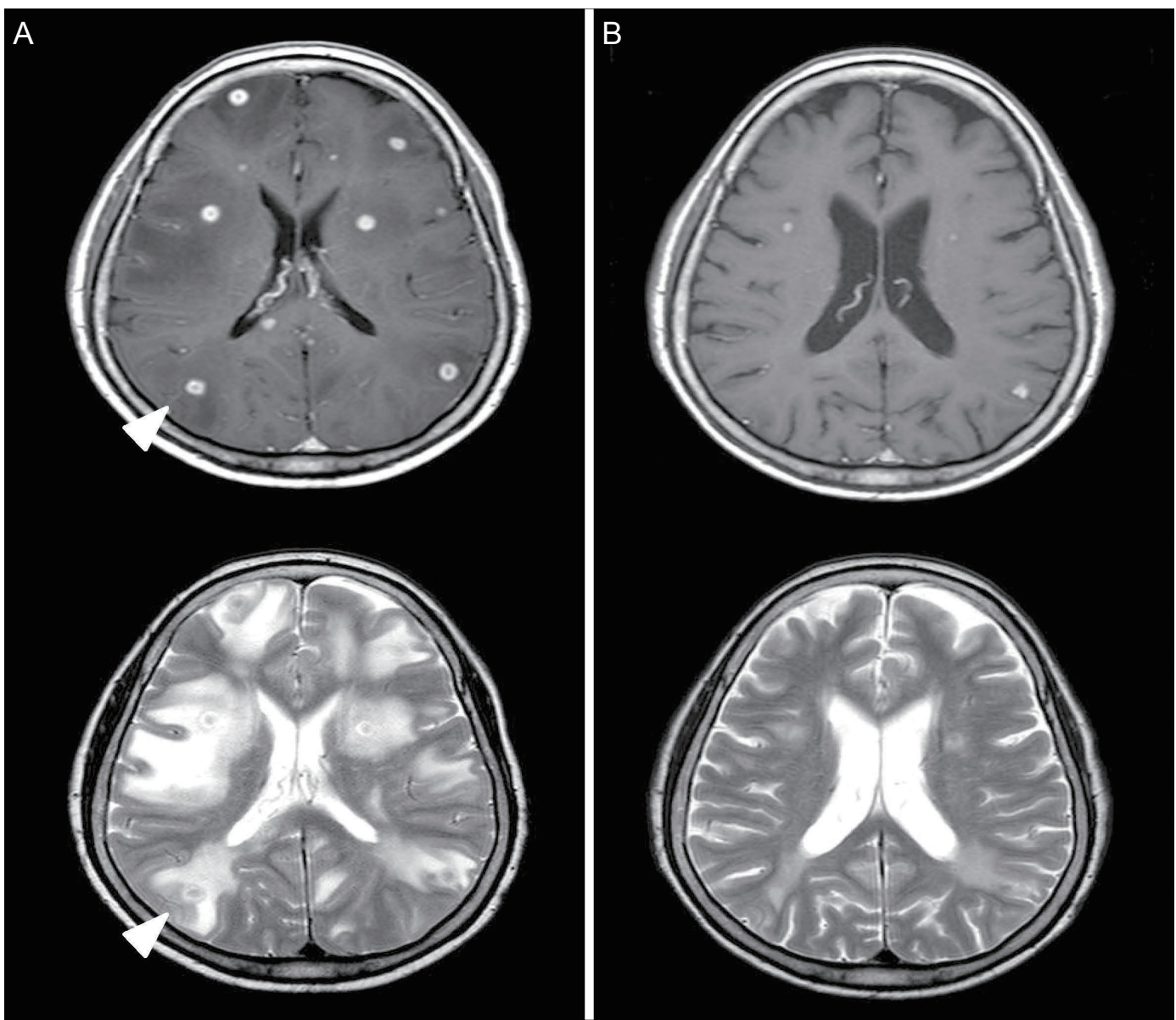

Figure 6. MRI of the brain on follow-up. (A) Follow-up brain MRI after one month of anti-tuberculosis therapy reveals no gross intervening change in the numerous small, ring-enhancing nodules. Several new lesions had developed (arrowheads). (B) Follow-up brain $\mathrm{MRI}$ at three months after symptom onset shows a significant decrease in the size and number of lesions and a marked amelioration of the surrounding edema. MRI, magnetic resonance imaging.

not diagnosed and treated early.,3

Although miliary TB has recently occurred more commonly in older than in younger people, in the past it occurred mainly in children. ${ }^{3}$ It appears to be a result of endogenous reactivation of $\mathrm{TB}$ and a consequent massive lymphogenous and hematogenous dissemination, ${ }^{3}$ and the widespread use of immunosuppressive, immunomodulating, and cytotoxic agents to treat various conditions in older people may be one reason for the shift in age-specific incidence. This shift can cause diagnostic confusion for clinicians because the radiological appearance of miliary TB can resemble that of metastatic cancer; there have been several reports that miliary TB mimics metastatic cancer. ${ }^{2,4}$ Furthermore, the efficacy of microbiological confirmation of miliary TB is unsatisfactory compared with that for pulmonary TB, despite the fact that miliary TB nearly always involves the lungs. It is known that only $30 \%$ to $65 \%$ of cases of miliary TB are positively diagnosed on the basis of culture of the pathogen from sputum. ${ }^{5}$ Bronchoscopic examination with combinations of BAL and TBLB would be expected to have a higher diagnostic yield. ${ }^{6}$

In our case, the patient was initially suggested, based on the radiological images, to have renal cell carcinoma with multiple metastases to lung and brain. However, the additional diagnostic approaches of $B A L, T B L B$, and renal biopsy revealed TB infection involving multiple organs, rather than a malignancy. As a result, our patient was finally diagnosed with miliary TB mimicking brain metas- 
tasis from renal cell carcinoma. The patient improved after a relatively short duration of anti-TB treatment. However, her neurologic symptoms unfortunately worsened, and follow-up brain MRI suggested the possibility of the concurrent existence of miliary TB and brain tumor. An additional diagnostic procedure of brain biopsy revealed no evidence of malignancy. Therefore, we concluded that the patient should be diagnosed with miliary TB with a subsequent paradoxical response to treatment, and continued with the anti-TB medication. The paradoxically enlarged intracranial tuberculomas had nearly resolved in the follow-up brain MRI.

A paradoxical response is defined as the clinical or radiological worsening of preexisting TB lesions or the development of new lesions not attributable to the normal course of disease, in a patient who initially improved with anti-TB therapy. ${ }^{7}$ A paradoxical response can occur in 10\% to $15 \%$ of TB patients who do not have concurrent HIV infection. ${ }^{8}$ The immunological components involved in a paradoxical response have not been fully described, but some studies suggest that it may be mediated by an exaggerated response to the release of mycobacterial antigen from dying organisms. ${ }^{8,9}$ One study suggested that patients whose treatment is complicated by a paradoxical response are more likely to have lower baseline lymphocyte counts, followed by a surge. ${ }^{9}$ However, unlike this previous report, our patient did not have relatively low baseline lymphocyte counts followed by a surge. In a recent prospective cohort study, predictors of paradoxical reaction were female gender, HIV positivity and a shorter duration of illness. ${ }^{10}$ Since our patient was female and had relatively short-duration of illness, these are considered to be consistent with previous findings. To reduce the paradoxical reaction, corticosteroid regimen consisted of intravenous dexamethasone for 4 weeks $(0.4 \mathrm{mg} / \mathrm{kg} / \mathrm{day}$ and then tapered off decreasing $0.1 \mathrm{mg} / \mathrm{kg}$ every week) and then oral treatment for 4 weeks, starting at a total of $4 \mathrm{mg}$ per day and decreasing by $1 \mathrm{mg}$ each week. ${ }^{10}$

A large number of infectious and non-infectious diseases can cause multiple brain enhancing lesions. In one prospective Indian study, TB was the commonest infective pathology, followed by neurocysticercosis. Neoplastic diseases were common non-infective causes. ${ }^{11}$ Therefore, it is important to distinguish TB granuloma from brain metastasis. MRI is generally considered to be superior to CT scan in assessing central nervous system (CNS) TB, and has been used in the diagnosis. ${ }^{12}$ However, CNS TB of MRI feature is sometimes non-specific, because it has other radiologic manifestation either diffuse form as basal exudative leptomeningitis or in a localized form as tuberculoma, abscess, or cerebritis. ${ }^{12}$ So, it may mimic other infectious or noninfectious etiology such as metastatic tumor. In our case, MR imaging of the brain showed multiple ring-enhacing nodules. In an attempt of differential diagnosis of multiple ring-enhacing nodules, Garg and Sinha, ${ }^{11}$ described some aspect of MRI feature (Table 1).

Although acid-fast staining of CSF is the most rapid method for detection of mycobacteria, this lacks sensitivity. Isolation of Mycobacterium tuberculosis from CSF can be insensitive if large amounts of CSF ( $\geq 2 \mathrm{~mL})$ are not

Table 1. Differential diagnosis between tuberculoma and metastatic brain tumor according to radiologic feature of $\mathrm{MRI}^{11}$

\begin{tabular}{|c|c|c|c|c|c|c|c|}
\hline & \multicolumn{7}{|l|}{ MRI } \\
\hline & $\mathrm{T} 1$ & $\mathrm{~T} 2$ & Enhanced & DWI & $A D C$ & MRS & MT ratio \\
\hline Tuberculoma & $\begin{array}{l}\text { Core iso- to } \\
\text { hypointense }\end{array}$ & $\begin{array}{l}\text { Core iso- to hypointense } \\
\text { with an iso- to } \\
\text { hyperintense rim }\end{array}$ & $\begin{array}{l}\text { Rim enhancement } \\
\text { irregular outline }\end{array}$ & Restriction & Low & Lipid peak & Lower \\
\hline Metstasis & Hypointense & $\begin{array}{l}\text { Hyperintense, may } \\
\text { involve the corpus } \\
\text { callosum }\end{array}$ & $\begin{array}{l}\text { Thick rim or } \\
\text { nodular } \\
\text { enhancement }\end{array}$ & Dark & High & $\begin{array}{l}\text { Suppressed NAA } \\
\text { and Cr. } \\
\text { Elevated choline } \\
\text { and lactate }\end{array}$ & - \\
\hline
\end{tabular}

MRI, magnetic resonance imaging; DWI, diffusion-weighted imaging; ADC, apparent diffusion coefficient; MRS, magnetic resonance spectroscopy; MT, magnetisation transfer; NAA, N-acetylaspartate. 
tested. ${ }^{13}$ In addition, although PCR has been reported to be relatively specific in most reports (82-100\%), its sensitivity has ranged from $32 \%$ to $100 \%{ }^{14}$ To distinguish miliary brain TB from brain metastasis, proton magnetic resonance spectroscopy (MRS) may also serve as a diagnostic tool. Neoplastic lesions have elevated Cho, decreased $\mathrm{Cr}$ and NAA, while non-neoplastic lesions such as cerebral ischemia and cerebral abscesses had markedly decreased in $\mathrm{Cho}, \mathrm{Cr}$, and NAA. Furthermore, lipids and lactate are normally not detectable in a healthy brain. The lipids detected by MRS reflect the degree of micro- and macronecrosis. The intracranial metastatic tumor showed relatively higher lipid levels than what is reflected in a non-metastatic brain tumor. However, in case of TB abscess or toxoplasmosis, this method has a limited application. ${ }^{15}$

In conclusion, clinicians should be aware that miliary TB, despite its rarity, can mimic metastatic cancer even in older people, especially in TB-endemic regions such as Korea. In addition, the possibility of a paradoxical response should also be suspected in patients who present with new neurological findings while on anti-TB therapy, because the occurrence of a paradoxical response is not uncommon.

\section{Conflicts of Interest}

The authors of this study have no conflict of interests to disclose.

\section{Acknowledgements}

This research was supported by Basic Science Research Program through the National Research Foundation of Korea (NRF) funded by the Ministry of Education (NRF2017R1D1A1B03029672)

\section{REFERENCES}

1. Centers for Disease Control and Prevention. Reported tuberculosis in the United States, 2012 [online]. Available at: https://www.cdc.gov/tb/. Accessed at April 5, 2018.

2. Greschus S, Kuchelmeister K, Oeynhausen S, Fischer HP, Ur- bach $\mathrm{H}$. Cerebral tuberculoma mimicking brain tumor. Clin Neuroradiol 2014;24:389-93.

3. Sharma SK, Mohan A, Sharma A, Mitra DK. Miliary tuberculosis: new insights into an old disease. Lancet Infect Dis 2005;5:415-30.

4. Ko KT, Na DJ, Han SH, Jung SS, Moon KM, Kim DJ, et al. Unusual presentation of miliary tuberculosis. Tuberc Respir Dis 2007;63:67-71.

5. Sharma SK, Mohan A, Sharma A. Challenges in the diagnosis \& treatment of miliary tuberculosis. Indian / Med Res 2012;135:703-30.

6. Burk JR, Viroslav J, Bynum LJ. Miliary tuberculosis diagnosed by fibreoptic bronchoscopy and transbronchial biopsy. Tubercle 1978;59:107-9.

7. Wasay M. Central nervous system tuberculosis and paradoxical response. South Med I 2006;99:331-2.

8. Breen RA, Smith CJ, Bettinson H, Dart S, Bannister B, Johnson $M A$, et al. Paradoxical reactions during tuberculosis treatment in patients with and without HIV co-infection. Thorax 2004;59:704-7.

9. Cheng VC, Yam WC, Woo PC, Lau SK, Hung IF, Wong SP, et al. Risk factors for development of paradoxical response during antituberculosis therapy in HIV-negative patients. Eur I Clin Microbiol Infect Dis 2003;22:597-602.

10. Singh AK, Malhotra HS, Garg RK, Jain A, Kumar N, Kohli N, et al. Paradoxical reaction in tuberculous meningitis: presentation, predictors and impact on prognosis. BMC Infect Dis 2016;16:306

11. Garg RK, Sinha MK. Multiple ring-enhancing lesions of the brain. J Postgrad Med 2010;56:307-16.

12. Rock RB, Olin M, Baker CA, Molitor TW, Peterson PK. Central nervous system tuberculosis: pathogenesis and clinical aspects. Clin Microbiol Rev 2008;21:243-61, table of contents.

13. Baker CA, Cartwright CP, Williams DN, Nelson SM, Peterson PK. Early detection of central nervous system tuberculosis with the gen-probe nucleic Acid amplification assay: utility in an inner city hospital. Clin Infect Dis 2002;35:339-42.

14. Lin IJ, Harn HJ, Hsu YD, Tsao WL, Lee HS, Lee WH. Rapid diagnosis of tuberculous meningitis by polymerase chain reaction assay of cerebrospinal fluid. / Neuro/ 1995;242:147-52.

15. Möller-Hartmann W, Herminghaus S, Krings T, Marquardt G, Lanfermann $\mathrm{H}$, Pilatus $\mathrm{U}$, et al. Clinical application of proton magnetic resonance spectroscopy in the diagnosis of intracranial mass lesions. Neuroradiology 2002;44:371-81. 